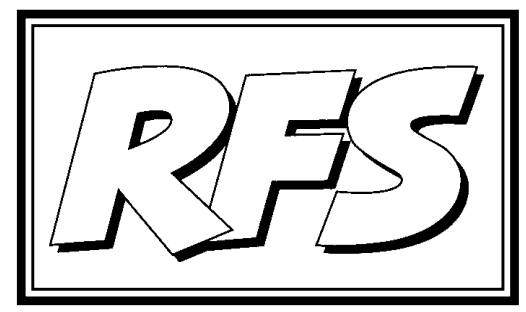

Revista de Fomento Social, 60 (2005), 387-405

\title{
Una reflexión sobre la construcción del Espacio Europeo de Educación Superior
}

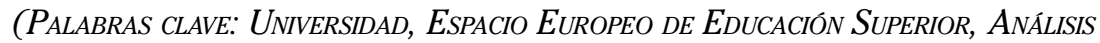
VALORATIVO.

KEY WORDS: UNIVERSITY, EUROPEAN SPACE FOR HIGHER EDUCATION, EVALUATION ANALYSIS)

\section{Introducción}

En el fragor de los debates surgidos en torno al tormentoso proceso de ratificación del texto de la Constitución Europea -muy cuestionada tras el rechazo sufrido en los referenda francés y holandés- podría extenderse entre los ciudadanos europeos la sensación de que "la construcción europea está en peligro". Aparte de los avatares, más o menos problemáticos, del proceso económico y político de construcción de la Unión Europea, hay otra serie de avances, que 
desbordan el marco jurídico estrictamente comunitario, y que suponen avances sustanciales en la línea de una mayor internacionalización y europeización de la cultura. Uno de ellos, de especial actualidad, es el avance hacia la creación de un Espacio Europeo de Educación Superior (EEES), también conocido como el proceso "de Bolonia", porque en esa ciudad italiana tuvo lugar el acuerdo básico por el que se puso en marcha.

Ciertamente, como veremos, el proceso llamado "de Bolonia", consistente en una armonización y modernización de las universidades europeas, no es un "producto" específico de la Unión Europea, pues las competencias sobre estas materias educativas no han sido transferidas a los órganos legislativos de la Unión. Se trata de una dinámica que resulta de convenios internacionales que desbordan los límites de la UE, pues, desde su inicio, participan en él países no pertenecientes todavía a la Unión. Ello no quita que estemos ante un movimiento que sólo se entiende en el marco de la creciente "europeización" y "globalización" de la cultura. Los europeos más jóvenes, en particular, han nacido y vivido en un entorno marcado por la creciente "integración supraestatal europea" no sólo de la economía, sino también de aspectos relevantes de la vida cotidiana. Para ellos es lo más natural poder "disfrutar" de logros que van desde la libertad de circulación e instalación de los europeos por los países de la UE, hasta la masiva participación en los programas de intercambios de estudiantes, pasando por la ya connatural circulación del euro como moneda única, sin olvidar la concertación y coordinación en políticas decisivas como la medioambiental o de investigación, etc.

Pues bien, la política universitaria es una de esas políticas en proceso de creciente coordinación. En muy pocos años, ha conocido avances espectaculares y, pese a su desconocimiento por la mayoría de la población, que no se siente directamente afectada por ella, puede suponer -y está ya suponiendo de hecho- una auténtica revolución en la manera de entender la enseñanza universitaria. Su impacto está siendo particularmente intenso en España, dado que será preciso introducir, en un tiempo relativamente corto, modificaciones sustanciales en nuestro sistema universitario de enseñanza-aprendizaje, que pueden significar un auténtico "shock" para muchos de los protagonistas de dicho sistema.

Dado que la RFS se publica en una institución universitaria y que, por ende, este asunto de la Convergencia Europea en Educación Superior ha sido objeto de repetidas reflexiones y análisis por parte de nuestro claustro de profesores, 
hemos decidido abordarlo en esta reflexión editorial, en un intento de provocar la reflexión y el diálogo ${ }^{1}$. Entre otras cosas, intentamos poner de relieve las implicaciones más profundas de los cambios en curso que tienen el peligro de ser considerados como meras adaptaciones técnicas y pedagógicas de cara a una mayor homogeneización del sistema europeo de educación superior.

Hemos estructurado nuestra reflexión en los siguientes apartados: comenzamos con una breve reseña histórica del proceso, subrayando los aportes específicos fundamentales de cada uno de los pasos dados a partir de la declaración de la Sorbona. A continuación resumimos brevemente los componentes de la propuesta, pensando en aquellos lectores menos familiarizados con este tema; seguramente las personas pertenecientes a la comunidad universitaria pueden omitir la lectura de esta parte, y darla por conocida. Viene a continuación lo que consideramos que es nuestra aportación reflexiva; una valoración sistemática $y$ un conjunto de inquietudes acerca de las implicaciones más relevantes de este proceso de cambio de modelo universitario.

\section{Breve reseña histórica del proceso}

La Convergencia Europea en Educación Superior (que también podríamos llamar el Espacio Europeo en Educación Superior nace por el convencimiento de que la universidad tiene un papel fundamental en la Sociedad del Conocimiento por lo que debe proporcionar un apoyo a la misma.

Su objetivo, por tanto, es conseguir la Calidad en educación superior a través de una modernización de los sistemas de enseñanza-aprendizaje. Ello llevará a una mayor Competencia respecto a los sistemas educativos de los países desarrollados, lo que a su vez, podría llevar a la atracción de estudian-

1 En otra ocasión abordamos un tema relacionado con éste. Véase: CONSEJo DE REDACCIÓN (1997), "Enseñar y aprender en la universidad", Revista de Fomento Social, no 206, abril-junio, pp. 133-155. Muchas de nuestras reflexiones de entonces resultan ser de actualidad en el marco del nuevo contexto al que dedicamos el presente editorial. Hemos contado para este texto con las reflexiones y el material del Encuentro Interuniversitario sobre este tema, organizado por UNIJES (Asociación de los Centros Universitarios de la Compañía de Jesús en España), celebrado en Madrid los días 22 y 23 de septiembre de 2005. Asimismo, han sido de gran utilidad las sesiones de formación sobre este tema tenidas en ETEA: el 8 de enero de 2005 bajo la dirección del profesor Miguel Valcárcel, de la Universidad de Córdoba, celebramos un Seminario sobre EEES y el 19 y 20 de mayo de 2005 bajo la dirección de la profesora María José Bezanill Albisua, de la Universidad de Deusto, unas Jornadas sobre la Convergencia Europea, con especial atención al Proyecto Tuning co-pilotado por dicha universidad. 
tes de otros países, sobre todo de Asia e Iberoamérica. Al mismo tiempo, la universidad debería ser un soporte del Mercado Laboral Único, para lo cual es imprescindible la Comparabilidad de los sistemas de educación superior. Esta Comparabilidad permitiría la movilidad de estudiantes, profesores y personal de administración y servicios.

El proceso de esta convergencia ha tenido distintos hitos:

- Programa ERASMUS que desde 1987 ha permitido el intercambio de alumnos.

- Declaración de la Sorbona (1988). Creación del EEES; marco de enseñanza-aprendizaje común; movilidad de personas; potenciación de las posibilidades de empleo.

- Declaración de Bolonia (1999)². Suplemento al título; sistema basado en grado y postgrado; sistema de créditos europeo (European Credits Transfer System: ECTS); movilidad; cooperación europea; dimensión europea en la educación superior ${ }^{3}$.

- Comunicado de Praga (2001). Refuerzo de los 6 objetivos de Bolonia; formación a lo largo de toda la vida (Lifelong learning); mecanismos de acreditación y certificación; calidad educativa e investigadora.

- Conferencia de Berlín (2003). Sistemas de calidad para 2005; sistemas de dos ciclos para 2005; marco europeo de titulaciones, reconocimiento de título y períodos de estudios; promoción de la movilidad; generalización del ECTS; protagonismo de la investigación, movilidad doctoral y postdoctoral, cooperación al nivel de doctorado.

2 Los países firmantes de esta declaración fueron: Austria, Bélgica, República Checa, Bulgaria, Estonia, Dinamarca, Francia, Finlandia, Alemania, Hungría, Grecia, Irlanda, Islandia, Letonia, Italia, Luxemburgo, Lituania, Luxemburgo, Países Bajos, Malta, Polonia, Noruega, Rumanía, Portugal, Eslovenia, Eslovaquia, Suecia, España, Reino Unido, Suiza.

3 Son seis los objetivos recogidos en la Declaración de Bolonia: A) La adopción de un sistema fácilmente legible y comparable de titulaciones, mediante la implantación, entre otras cuestiones, de un Suplemento al Diploma. B) La adopción de un sistema basado, fundamentalmente, en dos ciclos principales. C) El establecimiento de un sistema de créditos, como el sistema ECTS. D) La promoción de la cooperación Europea para asegurar un nivel de calidad para el desarrollo de criterios y metodologías comparables. E) La promoción de una necesaria dimensión Europea en la educación superior con particular énfasis en el desarrollo curricular. F) La promoción de la movilidad y remoción de obstáculos para el ejercicio libre de la misma por los estudiantes, profesores y personal administrativo de las universidades y otras instituciones de enseñanza superior europea. 
- Conferencia de Bergen (2005). Adopción de una estructura cíclica de titulaciones; reconocimiento de títulos y períodos de estudio; sistemas de evaluación de la calidad.

Por lo que respecta a la situación española, aunque la incorporación al proceso no se inicia hasta 2001, las etapas se han ido alcanzando en los plazos previstos. La aplicación, sin embargo, es más lenta que la elaboración de documentos, por lo que la puesta en marcha de las normas mantiene un cierto retraso.

Los textos básicos españoles a los que hay que hacer referencia en torno a esta cuestión son los siguientes:

- Ley orgánica de universidades (2001). Incorpora: suplemento europeo al título; adopción del sistema ECTS; ciclos y títulos oficiales; fomento de la movilidad.

- Acuerdo de la Asamblea General de la Conferencia de Rectores de las Universidades Españolas (CRUE) (2002). Extiende a España la declaración de Bolonia y su repercusión sobre la estructura de las titulaciones.

- Documento Marco del Ministerio de Educación, Cultura y Deporte (2003). Integra el sistema universitario español en el EEES.

- Real Decreto sobre el suplemento europeo al título (2003).

- Real Decreto sobre el sistema ECTS y el sistema de calificaciones en las titulaciones universitarias oficiales (2003).

- Real Decreto sobre estudios de grado (2005).

- Real Decreto sobre estudios de postgrado (master, doctorado) (2005). ${ }^{4}$

Asimismo, también existe un proyecto europeo, denominado Proyecto Tuning, que tiene especial importancia en España. Inició sus trabajos en 2001, coordinado por las Universidades de Deusto y Groningen. Su objetivo es el avance en la creación del EEES. Participan más de 100 expertos de siete áreas de conocimiento, procedentes de distintas instituciones de 16 países de la Unión Europea. La primera fase terminó en 2002 (competencias genéricas de la educación superior y de las distintas titulaciones, competencias específicas, concepto y enfoque del ECTS, nuevos métodos de enseñanza-aprendizaje,

4 En estos momentos se está elaborando una nueva norma en la que lógicamente se tratan estos temas. 
evaluación y calidad). La segunda fase finalizó en 2004 (profundización en los nuevos métodos de enseñanza-aprendizaje y evaluación y calidad, aplicación de la metodología a dos nuevas áreas, ampliación del proyecto a nuevos países, actuación como plataforma de intercambio de conocimiento y experiencias).

\section{Un resumen de las propuestas}

Son varios los componentes fundamentales de las reformas en curso, ya aprobadas y en fase de aplicación.

\subsection{Los ciclos universitarios}

La enseñanza universitaria se organizará en dos ciclos. El primer ciclo, que dura como mínimo 3 años, da derecho a un diploma que será utilizable como cualificación en el mercado laboral europeo. El segundo ciclo, permite obtener un título de master, tras uno o dos años de enseñanza, cuyo contenido corresponde a una formación avanzada, de carácter especializado o multidisciplinar, dirigida a una especialización académica, profesional o de inicio a la investigación. Existirá también un tercer ciclo que está constituido por los estudios de doctorado, con una duración de dos años, que junto a la elaboración y defensa de la tesis doctoral dará lugar al título de doctor.

3.2. El crédito europeo y los cambios en el sistema de enseñanza-aprendizaje

El aspecto del EEES que se ha subrayado con más frecuencia es el establecimiento de un sistema de créditos: el crédito europeo. No hay que olvidar, sin embargo, que este concepto incorpora un nuevo enfoque de la enseñanza universitaria, no es sólo una nueva unidad de medida de la docencia.

El crédito europeo (el varias veces citado ECTS) "es la unidad de medida del haber académico, que representa la cantidad de trabajo del estudiante para cumplir los objetivos del programa de estudios... conducentes a la obtención de títulos universitarios de carácter oficial y validez en todo el territorio nacional" (Real Decreto 1125/2003).

El sistema de enseñanza-aprendizaje que va unido al ECTS no es absolutamente novedoso ya que existen experiencias anteriores en algunos países. Quizás, lo más significativo es la generalización de este sistema a todo el EEES y sobre todo la medida de la actividad académica desde el punto de vista del 
trabajo del alumno y no del contenido académico, es decir la actividad del docente.

El crédito que se ha venido utilizando en España corresponde a 10 horas presenciales. El crédito europeo corresponde a 25-30 horas de trabajo del estudiante; un curso escolar corresponde a 60 créditos que se desarrollan durante 36-40 semanas; se deduce que el trabajo de un curso escolar oscila entre 1.500 y 1.800 horas de trabajo del estudiante. Estas horas de trabajo se clasifican en horas presenciales (clases teóricas y prácticas, seminarios, resolución de problemas, estudio de casos, proyectos, tutorías, evaluaciones continuas y examen final) y horas de trabajo personal (preparación de apuntes, búsqueda de materiales, lecturas, preparación de exámenes, redacción de trabajos, realización de informes, estudios empíricos, etc.). La distribución entre horas presenciales y horas de trabajo personal podrá lógicamente ir evolucionando a lo largo del desarrollo de una carrera: así, los alumnos del primer curso tendrán, quizás, un 60-70\% de clases presenciales y un 40-30\% de horas de trabajo personal; en los últimos cursos esta distribución cambiaría sustancialmente: 20-30\% de clases presenciales, frente a 80-70\% de trabajo personal.

El sistema ECTS garantiza un nivel de formación comparable y la equivalencia de valor formativo y asegura la transparencia de los sistemas universitarios. Asimismo, permite la movilidad de estudiantes, del personal docente y del administrativo, el reconocimiento mutuo, el mercado laboral único y la atracción de estudiantes no europeos.

El modelo para el desarrollo de la enseñanza-aprendizaje, que está implícito en el sistema ECTS, comprende entre otros los siguientes aspectos: aprendizaje de competencias (lo que el titulado es capaz de hacer y lo que es capaz de seguir aprendiendo); aprendizaje autónomo; trabajo en equipo; consideración de los valores; evaluación de las actitudes (responsabilidad del estudiante ante su propio aprendizaje).

\subsection{El suplemento europeo al título (suplemento al diploma)}

Es un documento anejo al título que contiene un sistema común de acumulación y transferencia de créditos y calificaciones que permitirá una lectura fácil en todos los Estados para facilitar la libre circulación de profesionales en la Unión Europea.

El documento ha sido desarrollado por la UE, el Consejo de Europa y la UNESCO. Su objetivo es lograr la transparencia y reconocimiento académico 
y profesional en aras de la Comparabilidad. Contendrá datos del estudiante, centro, estudios realizados... Con el fin de alcanzar la Comparabilidad todas las asignaturas cursadas deberán referirse a créditos ECTS y notas del sistema europeo (ECTS grades). Hay un proyecto de modelo informático, elaborado por las universidades de Deusto, Valencia y Minho, que permite reproducir y traducir en un segundo idioma comunitario el expediente académico.

Los ECTS grades permiten una conversión rápida e inmediata de las calificaciones nacionales para permitir su lectura en todos los países de Europa.

En resumen, en las reformas contenidas en el EEES aparecen algunos elementos y a conocidos: ciertos aspectos de la universidad americana, otros del mundo universitario británico e incluso se citan a veces los modelos universitarios medievales. No obstante, los cambios, sobre todo en la Europa continental, son muy profundos, puesto que llevarán probablemente a un nuevo sistema, casi desconocido hasta ahora. A esto unimos la rapidez con la que se quiere poner en marcha el nuevo modelo, por lo menos en España. Subrayamos asimismo la ausencia de normas europeas propiamente comunitarias ${ }^{5}$, ausencia que se cubrirá con la aprobación en cada país de una legislación propia que no tiene por qué coincidir en todos los miembros de la UE. En resumen, todas estas circunstancias llevan a un elevado nivel de incertidumbre que hace difícil la valoración del proceso y el análisis de los efectos de su puesta en marcha. No obstante, hecha esta breve exposición del proceso en marcha y de sus aspectos más relevantes, ofrecemos a continuación una valoración del mismo ${ }^{6}$.

\section{Una valoración sistemática del proceso}

Después de esta presentación de lo que quiere ser la reforma de la universidad propuesta en el proyecto del EEES, es conveniente hacer algunas reflexiones valorativas que nos ayuden a situar Bolonia y a identificar las oportunidades y amenazas que todo el proceso representa para la universidad europea.

5 Nos referimos al hecho-al que aludíamos en la introducción-de que estamos ante un proceso que resulta de convenios internacionales; no es un "producto" específico de la UE, pues las competencias sobre estas materias no han sido transferidas a los órganos legislativos de la Unión.

6 Aunque aludimos continuamente a un espacio europeo, nuestros comentarios se concretarán principalmente al caso español. 


\subsection{Una universidad centrada en el alumno}

Aunque pueda parecer que toda universidad responde a este perfil, la realidad no siempre es así. El profesor ha sido normalmente el centro de la enseñanza superior; el protagonismo de la investigación, que evidentemente es indispensable en toda universidad, e incluso de las actividades de extensión, ello explica que a veces la "clase", en general, y el alumno en particular, hayan tenido un papel secundario. En la universidad privada ha sido frecuente que el gestor haya tenido quizás mayor importancia, ya que la ausencia de recursos públicos ha puesto en un primer plano la necesidad de cubrir los gastos, para lograr el equilibrio de sus cuentas.

Hoy se dice que la institución universitaria ha ido evolucionando desde una universidad centrada en el docente hacia una universidad centrada en el administrador. Esto significa que el estudiante siempre ha ocupado un segundo plano. "Centrada en el docente" implica estar pensada y estructurada desde las estructuras del sabery de las exigencias de quienes se sienten profesionales del mismo; menos atenta, por tanto, a las necesidades del estudiante quien, por lo general, busca en el saber sólo un instrumento para su desarrollo profesional. "Centrada en el administrador" significa convertida en empresa, con todas las ventajas e inconvenientes que ello conlleva. Ventajas, en términos de racionalidad y búsqueda de la eficiencia de unos recursos humanos y materiales. Inconvenientes, siempre que la racionalidad económica se erija en un fin que impone su férrea ley sobre lo que es propio de toda universidad, la docencia y la investigación.

Estamos, por tanto, ante un cambio radical, que se indica reiteradamente en las declaraciones del EEES. Como ya se ha indicado, la creación del crédito europeo va en esta dirección: su aplicación se basa en el trabajo del alumno y no exclusivamente en el del profesor. De la misma forma, la graduación de las enseñanzas en el proceso de aprendizaje va en el mismo sentido.

Como es lógico, este cambio no es solamente una nueva forma de evaluación, sino que exige una adecuación de la enseñanza a este objetivo: reducción de clases magistrales, incremento de otras actividades (trabajo personal, trabajo en grupo...), importancia de las tutorías. Todo ello precisa de una adaptación de los docentes, y de los alumnos, a un nuevo sistema, si se pretende alcanzar los objetivos de Bolonia.

En otros términos: ¿qué papel se le exige al profesor?; da la sensación que tiene que ser un "GEO", un comando especial: se le pide que sea un buen do- 
cente, que asesore, que sepa psicopedagogía... Quizás se está planteando un esquema utópico y, en algunos momentos, irreales.

De todo lo anterior habría que subrayar la necesidad de una formación previa del profesorado en técnicas pedagógicas, tan escasa con frecuencia en las carreras universitarias, y también en la aplicación de los principios establecidos en las distintas declaraciones arriba citadas. Además, será preciso tenerlos en cuenta en las directrices de los planes de estudios y en su aplicación, de forma que la enseñanza se adapte al ritmo de aprendizaje del alumno. No hay que olvidar tampoco que este sistema puede dar lugar a resistencias por parte de los alumnos que observarán que les exige un mayor esfuerzo.

Por otra parte, este sistema "exige" que al diseñar los contenidos no se programe por asignaturas, sino por bloques de asignaturas o ámbitos de conocimiento. Ello conlleva una colegialidad del profesorado a la hora de programar-desarrollar-evaluar, ya que la programación por asignaturas llevaría a una atomización de competencias o a un duplicado de éstas. Es decir, las asignaturas no sólo se deberán agrupar en áreas de forma nominal, sino que deberá realizarse una programación y evaluación conjunta de cada área, lo cual puede llevar a una especie de "guerra" entre profesores...

Por el contrario, nos tememos que los resultados puedan ser parecidos a lo que ocurre en Secundaria (consecuencias de determinados proyectos de leyes de educación): desmotivación del profesor y "balcanización”, cada uno se dedicará a lo suyo y lo hará lo mejor que pueda. Aquí la teoría irá por un lado y la práctica real por otro. Paradójicamente, el "proceso" requiere que el alumno haya alcanzado una buena formación en secundaria, lo cual no está nada claro en estos momentos; pero esto es un problema ajeno a la universidad, en el que no vamos a entrar.

Por último, es obvio que este sistema requiere medios adecuados: recursos informáticos, publicaciones y materiales (no sólo los típicos apuntes), clases y espacios adecuados al trabajo en equipo, profesores y tutores, como ya hemos indicado; y, lógicamente, recursos financieros suficientes.

\subsection{Homogeneidad de los estudios en el contexto europeo}

La transparencia de los estudios en cada país, la comparabilidad de los expedientes y la homogeneidad de los contenidos tienen ciertas finalidades: la movilidad de profesores, alumnos y, en último término, de los ciudadanos en el espacio europeo; la elevación del nivel de la universidad europea para que 
sea reconocida a nivel mundial; $y$, finalmente, el avance hacia una cooperación universitaria internacional.

En esta línea van las propuestas de diploma europeo (suplemento europeo al título), la propia definición del crédito europeo, el Marco Europeo Común de referencia para los idiomas, etc.

Este objetivo puede establecer ciertas contradicciones con otros principios, tales como la competitividad, la especialización y la autonomía de los Estados, lo que exige una armonización de dichos objetivos, para hacerlos compatibles, considerando que ninguno tiene prioridad sobre los demás.

\subsection{Competitividad y adaptación a las exigencias del mundo actual}

Esto se puede entender en un doble sentido: a) competencia entre las universidades europeas para prestar, en mayor medida, los servicios que demanda la sociedad; b) competencia de los centros europeos de enseñanza con los de otros países (EEUU etc.) para recuperar el papel que la cultura europea tuvo en otros tiempos.

Esta adaptación se puede explicar de varias formas:

A) Atender a los retos de la sociedad de la información (nuevas tecnologías, capacitar al alumno para que sea capaz de aprender por sí mismo, dada la rapidez en la evolución del conocimiento). Es preciso incorporar la innovación científica y tecnológica a las aulas y, en concreto, en términos instrumentales, las tecnologías de la información y de la comunicación; en este último sentido, se debe evolucionar a una nueva forma de relación de profesores y alumnos a través de estos medios, que no deben ser meramente una "plataforma" para comunicar apuntes. La adaptación mencionada tiene como condición la flexibilidad (planes de estudio, etc.), lo que, sin embargo, puede crear algún problema con la homogeneidad en el espacio europeo.

B) Atender a las necesidades del mercado de trabajo. Hay que conocer las necesidades reales de las empresas, para lo cual es muy conveniente "oír" a los antiguos alumnos y profesionales en general; en cuanto a los estudiantes actuales, la realización de prácticas en empresas puede facilitarles este conocimiento del mercado. Ahora bien, las necesidades del mercado en cuanto a sus cuadros, dirigentes, técnicos... deberá determinar la relación de las titulaciones ofertadas. 
Pero esta lógica adaptación al mercado no está libre de riesgos. Aunque la formación de profesionales es uno de los fines de la universidad, ello puede conducir fácilmente a valorar los centros por el éxito profesional de sus titulados ${ }^{7}$. Se ha dicho que podemos consolidar un modelo de universidad-empresa, en el que las instituciones que se plieguen a las exigencias del mercado serán las mejor valoradas y las que consiguieran recursos de todo tipo. Ello es especialmente significativo en las universidades privadas en las que el número de alumnos y la "rentabilidad" determinarán su permanencia en el sistema.

La polémica sobre el mantenimiento de las carreras de humanidades y de historia del arte en España es un ejemplo de este problema. Se trata de una cuestión de dos caras: la sociedad y el mercado dicen lo que les interesa o lo que sobra, pero la universidad no puede ser únicamente una empresa que se mueve por aquellos criterios exclusivamente. La insistencia de las declaraciones del EEES en la competitividad de la economía europea y en el papel de la universidad en este sentido, nos hace temer que el criterio del mercado es el que se pretende establecer en la universidad europea. Ello puede comportar un "abandono" de ciertos saberes de contenido más humanista e integral (filosofía, historia, etc.), indispensables para conocer el alcance que el saber en general tiene con respecto a la persona y a la sociedad.

C) Las exigencias de la globalización. Un mundo globalizado requiere una universidad abierta y flexible; sólo las instituciones con estas características pueden aspirar a su permanencia en el sistema. Para ello es necesaria la movilidad de los docentes, de los no docentes y de los propios estudiantes, movilidad a la que ya hemos aludido. Asimismo, la flexibilidad de los planes de estudio, es decir, su adecuación a las necesidades cambiantes de la sociedad, es también un requisito esencial, aunque ello pueda crear algún problema con relación a la homogeneidad en el espacio europeo.

7 A este respecto, véase la reflexión autocrítica de hace diez años de la Asociación de Universidades Jesuitas de América Latina (AUSJAL): "Llevamos décadas formando profesionales generalmente exitosos en sociedades fracasadas y cada vez más deshumanizadas. Nuestros egresados ocupan puestos de alta responsabilidad en las empresas privadas y en actividades gubernamentales. Sin caer en acusaciones panfletarias debemos, sin embargo, preguntarnos sobre las causas de esta disparidad entre el éxito individual de muchos de nuestros egresados y el naufragio de nuestras sociedades". (AUSJAL, (1995), Desafíos de América Latina y propuestas educativas, n. 69). 


\subsection{La evaluación de la Universidad}

Se trata de un aspecto muy relacionado con el anterior. Aunque no haya sido establecido inicialmente por el EEES, va unido a éste. La evaluación de centros, titulaciones y enseñantes es un fenómeno reciente que ha puesto en "ebullición" ciertas inercias de la universidad española; es algo positivo, por tanto, pero que también tiene sus riesgos.

Así, las agencias de evaluación (ANECA en el caso español) pueden priorizar los resultados de mercado a los que hemos aludido en el apartado anterior. Se piensa, y hay datos para ello, que las agencias pueden valorar los méritos pero también pueden establecer un dirigismo, de forma que determinadas escuelas $e$ incluso determinadas especialidades sean las que consigan la evaluación positiva. También se teme que la valoración, por una parte, y la competitividad, por otra, conduzcan a una universidad cuyos objetivos vengan fijados por el corto plazo.

Las agencias de evaluación valoran sobre todo la especialización. Se trata de una tendencia de la universidad moderna, anterior por tanto al modelo de Bolonia. Los principales elementos de ésta, recogidos anteriormente, van probablemente en esta línea de especialización. La complejidad de la ciencia actual la hacen inevitable, pero la ausencia de la figura del humanista que era el centro de la universidad medieval y moderna la empobrece y la deja sin algunos de los aspectos básicos universitarios. Volvemos de nuevo a las exigencias del mercado: ¿puede ser un centro universitario un mero impartidor de títulos? ¿realmente se elabora ciencia en la mayor parte de los centros actuales?

En efecto, el profesional que sale de nuestro sistema educativo suele ser un gran experto en una cosa y un ignorante en todo lo demás. Su tentación de proyectar esta visión que le da su especialización sobre toda la existencia no es pequeña. $Y$ tampoco es extraño ver su tendencia a resolver problemas complejos, como son todos los problemas importantes de nuestro mundo, desde perspectivas simplificadoras, las que le suministra inevitablemente su formación especializada. Y el simplismo deriva, o en un fuerte dogmatismo o en un relativismo total. No queremos negar con esto la necesidad de especializarse, pero si queremos formar personas hay que complementar esa formación para no generar tipos humanos empobrecidas.

Más aún, ¿no tienen el peligro de derivar hacia esta competencia mercantil los mismos sistemas de evaluación positiva y acreditación del profesorado? No puede negarse que un sistema que garantice la calidad del profesorado 
es esencial a la universidad. Pero si la calidad se reduce a ciertos criterios formales que hay que conseguir a toda costa, y a ser posible en el plazo más breve, estamos mercantilizando también la calidad: en el sentido técnico de la palabra "mercancía", de un bien que se aprecia más por su valor de cambio que por su valor de uso (no por lo que vale en sí, sino porque sirve para otra cosa, como puede ser el conseguir un puesto estable de trabajo). Ahora bien, si algo es consustancial a la universidad son la docencia y la investigación, y éstas no pueden reducirse a mercancías, porque tienen otros ritmos y otras exigencias...

Además, no es el principio de competencia el mejor criterio para distribuir bienes sociales. Los resultados los estamos viendo, más exacerbados en los países donde el modelo empresarial marca con más fuerza a la universidad. La competencia lleva a una especialización excesiva, y ésta no carece de contraindicaciones.

Tales inconvenientes se agravan si este modelo de universidad-empresa se deja llevar, como es hoy dominante en el mundo empresarial, por el "cortoplacismo": porque el objeto de la universidad, por su propia naturaleza, exige un tratamiento a medio y largo plazo.

Algo de esto podríamos ver en la aprobación de nuevas titulaciones, donde no siempre se tiene en cuenta la evolución de la ciencia, sino más bien las carreras que tienen éxito, a veces por poco tiempo, e incluso la "moda". No creemos que en esto deba consistir la flexibilidad universitaria, bien entendida.

En cierto modo, la evaluación podría o bien potenciar la flexibilidad o bien representar un "corsé" para los centros. La falta de experiencia en cuanto a los procesos de evaluación exige una maduración de las agencias para que verdaderamente sean un impulso de los fines del EEES.

\subsection{El alumno universitario}

La masificación universitaria (ahora en crisis por el descenso demográfico) ha tenido probablemente más aspectos positivos que negativos, pero ha llevado sin duda a un descenso del nivel del universitario. Estos eligen sus estudios, con frecuencia, en función de las "salidas" que les ofrecen las distintas titulaciones. El estudiante con ansias de aprender, con inquietud sobre el conocimiento, es claramente minoritario. En este sentido, el modelo del EEES podría elevar la formación de los discentes, pero ello no es seguro; hemos comentado los riesgos de mercantilización de la universidad, lo que no indica de forma terminante 
una enseñanza más "universitaria", incluso si se establecen unos métodos de enseñanza más efectivos.

El esquema de un primer ciclo impartido por todos los centros, que va orientado a atender las demandas del mercado de trabajo, y un segundo ciclo más minoritario, que proporcionará una formación más universitaria en el sentido tradicional tiene sus riesgos. Un sistema tan marcado por la competencia está llevando a un cierto darwinismo universitario, que la importancia creciente de los postgrados en el futuro tenderá a incrementar. Es lo que ocurre cuando el acceso a este segundo ciclo depende de los recursos económicos de los estudiantes. Entonces una buena formación especializada sólo estará al alcance de las clases más adineradas. ¿O no son los master más caros los que más se valoran luego en los curricula y en el mercado de trabajo? Pero esto afianza un modelo universitario donde el principio de justicia distributiva apenas tiene vigencia: llegarán a ser grandes profesionales no los más dotados, sino los más adinerados.

\subsection{La relación con la sociedad}

Este aspecto, muy relacionado con el anterior, también podría ser valorado positiva o negativamente; sólo la puesta en marcha del proceso dará una respuesta clara a este nuevo interrogante.

Aparte de la formación de profesionales y de la investigación científica, principales objetivos de la universidad, ésta presta además un doble servicio a la sociedad: a) realiza actividades de extensión, por medio de las cuales transmite conocimientos; b) analiza y estudia los problemas que preocupan a la sociedad no sólo en el terreno de las ciencias llamadas "duras", sino también en las cuestiones socioeconómicas.

La preocupación por el alumno, la competitividad y las necesidades del mercado son cuestiones prioritarias, pero que pueden dejar a un lado las obligaciones de la universidad con la sociedad. El EEES puede ser una oportunidad para que la universidad atienda a las necesidades de la sociedad, pero también puede ser una ocasión perdida.

\section{Algunos motivos generales de inquietud}

Además de las reflexiones críticas relativas a los componentes concretos del EEES contenidas en el apartado anterior, ofrecemos a continuación algunos aspectos preocupantes del modelo general. 


\subsection{El modelo de universidad}

Es bueno recordar que la universidad es una institución que nació en Europa, concretamente en la Europa medieval. Ya en su origen hay dos modelos de universidad que los historiadores se han encargado de identificar. En el llamado modelo de Bolonia, que fue el que se implantó también en España, los estudiantes contrataban a los maestros, los cuales vivían en colegios separados (era la universidad de los estudiantes). En el modelo de París, por el contrario, la iniciativa era de los profesores, que ofrecían sus servicios a los estudiantes por un precio; también en los colegios, que eran mixtos, todo el control lo ejercían los profesores.

En el transcurso de los siglos que nos separan de esos orígenes la institución universitaria se ha extendido por los cinco continentes, de manera que hoy existen universidades en (casi) todos los países del planeta. La universidad ha dejado de ser exclusivamente europea para convertirse en una institución universal. Evidentemente esta universalidad conlleva una inevitable variedad. ¿Será capaz Europa de mantener una impronta propia para la universidad de nuestro continente? No sabemos con toda seguridad si la "nueva" universidad surgida del proceso de Bolonia irá en esta dirección, pero ciertamente ése es el modelo que nosotros defendemos.

\subsection{Una visión de la universidad}

Tanta preocupación por el mercado de trabajo nos hace temer que estamos consolidando subrepticiamente el modelo de universidad-empresa. En esto haríamos un flaco favor a nuestra sociedad, que tiende tan alarmantemente a identificarse con el mercado. Porque ¿no estamos convirtiendo la lógica del mercado en la única lógica que gobierna todo el funcionamiento de nuestra sociedad? La universidad sería una institución más de las muchas que se ven obligadas a plegarse a tales exigencias. Desgraciadamente asistimos hoy a un escenario en el que la ley de la competencia preside la formación de los futuros profesionales e incluso marca todo su funcionamiento como institución.

Porque la universidad no es sólo un instrumento al servicio de las demandas más urgentes de la sociedad, las del mercado de trabajo ${ }^{8}$. Las relaciones entre

8 ¿Es el sistema anglosajón el único modelo de referencia? Hace unos años se ha suscitado en Estados Unidos un debate sobre el modelo pedagógico que crea "idiotas habilidosos", personas muy especializadas en cosas concretas pero "analfabetos" respecto a todo lo demás. 
ambas instituciones hay que enfocarlas más bien como interacción recíproca. La universidad nunca habrá de renunciar a ser "cerebro pensante de la sociedad", como gustaba repetir Ignacio Ellacuría, el malogrado rector de la Universidad Centroamericana de San Salvador. Y ser cerebro pensante implica ser también instancia crítica. Su relación con la sociedad no es meramente pasiva (de respuesta a las demandas), sino activa (de cuestionamiento de esas mismas demandas y de las estructuras que subyacen a ellas).

\subsection{El centro de atención de la universidad}

Volviendo a un tema que ya enunciamos más arriba, cabe preguntarse: ¿es posible pasar de una universidad centrada en el docente o en el administrador a una universidad centrada en el estudiante? Parece que eso es lo que pretende el EEES. La insistencia en un modelo orientado, no a la transmisión de conocimientos, sino al aprendizaje (el ECTS) va en esa dirección. Lo mismo podría decirse, en principio, de su preocupación por responder a las demandas del mercado de trabajo: se busca preparar mejor a los futuros profesionales de nuestra sociedad.

Nos contentaríamos, por tanto, si la universidad que se vaya configurando en el proceso que arranca de la declaración de Bolonia, buscase una buena articulación de los tres agentes que hemos mencionado: el docente, el estudiante y el administrador. La tarea no será fácil. Prueba de ello es que en la historia nunca se ha alcanzado satisfactoriamente esta síntesis. Porque hay que luchar contra el corporativismo docente, contra el pragmatismo del estudiante y contra el economicismo del administrador: y sabemos con qué fuerza arraigan estas tendencias en los tres colectivos mencionados, especialmente hoy.

\subsection{Los valores}

No es correcto plantear si el EEES puede ir unido a unos valores, o si se basará en la ausencia de ellos. Una institución de este tipo defiende o presenta unos ciertos valores en todo caso. Nuestra duda es si esta universidad va a estar centrada en los problemas de las personas, en la cooperación (incluso en el sentido que se la da hoy a esta palabra), en la justicia, en la democracia..., o si bien sus valores van a ser la competitividad (que se ha comentado anteriormente) y el mercado, en el sentido de la globalización.

Por otra parte, una universidad marcada por una competencia desenfrenada en su funcionamiento y en la orientación de su docencia y de su investigación

\section{EDITORIAL}


¿qué valores va a transmitir a los estudiantes que la frecuentan? Evidentemente, la competencia como eje vertebrador de la existencia humana y como valor prioritario del comportamiento social. Si es necesario asumir también esa otra dimensión de la docencia que implica transmisión de valores, ¿con qué facilidad podrán transmitirse valores de otra índole? El modelo mismo es el que está imponiendo sus propios valores, y todo lo demás será superestructura o voluntarismo estéril.

Esto obliga a la universidad a preguntarse continuamente: ¿en qué sociedad vivimos? ¿en qué sociedad queremos vivir? Y la respuesta a estas preguntas se proyectará indefectiblemente sobre la docencia, si ésta es algo más que aprendizaje de habilidades técnicas. No se trata de formar sólo especialistas, incapacitados para ver el mundo y sus problemas si no es desde la estrecha perspectiva de su especialidad. Formar personas, capaces de pensar y de preguntarse por las cuestiones últimas que legitiman lo concreto de cada día en la profesión, es tarea ineludible. Renunciar a la formación polifacética del hombre renacentista (hoy impensable a todas luces) no puede conducir a una universidad marcada por el mercantilismo "cortoplacista" que produce sólo especialistas útiles para mantener los engranajes de nuestra sociedad tecnificada y pragmática.

\section{Conclusión}

Como decíamos al principio, la amplitud y profundidad del cambio proyectado es una causa de la incertidumbre sobre el futuro del EEES. La rapidez de su aplicación (debe completarse en 2010) ${ }^{9}$ tiende también a aumentar la incertidumbre; en concreto, en el caso de España, el retraso de la implementación en los foros europeos va a hacer más arriesgada la apuesta por este modelo.

Una última cuestión crítica: ¿el EEES tiene como objetivo el modelo de universidad estadounidense o el de la universidad europea? No es claro si existe un modelo universitario europeo y en qué consiste este último. Se dice que la universidad europea, en comparación con la de USA, es menos darwinista, menos especializada, más humanista y más global y universal ${ }^{10}$.

9 Aunque, dado el gran número de países que lo han suscrito (véase nota 2 supra), de su distinto nivel de desarrollo relativo, y de que cada uno va a ir al ritmo que le permitan sus estructuras administrativas y universitarias, hacen prever un escalonamiento temporal significativo en la puesta en marcha del EEES por parte de los distintos países.

10 Lo que no dejaría de ser sino una concreción de la diferencia entre los dos modelos de capitalismo dominante: el renano y el sajón. Véase Michel AlBerT, (1993), Capitalismo contra 
Como resumen podemos afirmar que los problemas de la universidad vienen de más lejos. Bolonia nos ha ayudado a ponerlos todos ellos sobre la mesa. Se lo tenemos que agradecer y aprovechar las oportunidades que nos da para iniciar ciertas reformas. En este sentido Bolonia ha sido un motor. Pero también puede ser (está siendo ya) un corsé, en la medida en que nos conduzca hacia un modelo incompleto de universidad, aparentemente orientada al alumno, pero reducida a una empresa y sometida a la lógica mercantil, nada crítica con nuestra sociedad, en modo alguno "cerebro pensante" de la misma.

capitalismo, Ed. Paidós, Estado y Sociedad, $2^{\mathrm{a}}$ edición. A este respecto nos ha iluminado mucho la lectura de la obra SheIla SLAUghter AND LARRY L. LesLie, (1997), Academic Capitalism. Politics, Policies and the Entrepreneurial University. Baltimore and London. Ed. John Hopkins. El libro trata de lo que los autores denominan "marketización" de la academia. Se trata de un análisis riguroso, muy documentado, de la evolución de la universidad en el mundo anglosajón, con especial referencia a Estados Unidos. Sólo algunas citas bastan para hacer visible en qué línea van sus conclusiones más relevantes: "el profesorado y las instituciones se orientan a competir o a aumentar su competencia por fondos externos" (p. 209). "Esos dólares externos habitualmente están "amarrados a investigación relacionada con el mercado" (p. 209). "...para promover la formación que directamente satisface las necesidades de los negocios y de la industria" (p. 213). "...el dinero se trasladó hacia las ciencias naturales y la ingeniería y se apartó de las ciencias sociales y de las humanidades" (p. 245)."Los profesores a dedicación exclusiva en campos que estén lejos del mercado tendrán poco tiempo para la investigación y los trabajos becados". 